\title{
Set Theory and Structures
}

\author{
Neil Barton* and Sy-David Friedman ${ }^{\dagger}$
}

25 April $2018^{\ddagger}$

\begin{abstract}
Set-theoretic and category-theoretic foundations represent different perspectives on mathematical subject matter. In particular, category-theoretic language focusses on properties that can be determined up to isomorphism within a category, whereas set theory admits of properties determined by the internal structure of the membership relation. Various objections have been raised against this aspect of set theory in the category-theoretic literature. In this article, we advocate a methodological pluralism concerning the two foundational languages, and provide a theory that fruitfully interrelates a 'structural' perspective to a set-theoretic one. We present a set-theoretic system that is able to talk about structures more naturally, and argue that it provides an important perspective on plausibly structural properties such as cardinality. We conclude the language of set theory can provide useful information about the notion of mathematical structure.
\end{abstract}

\section{Introduction}

Two approaches (and, as the current volume shows, maybe more) in current debates on foundations provide radically different perspectives on mathematical subject matter. The set-theoreti ${ }^{1}$ perspective holds that all mathematical objects may be modelled in the sets, a formalism given in terms of a primitive membership-relation, and that this (in some sense) provides a useful foundation. The category-theoretic perspective, on the other hand, holds that all mathematical objects may be modelled by the kinds of mapping properties they have with respect to others. As we shall see below, the two provide somewhat different perspectives on mathematics. In light of this datum, a question which has sprung up in the literature is which foundation we should use for interpreting mathematics (assuming that a foundation is desirable at all).

\footnotetext{
* Kurt Gödel Research Center for Mathematical Logic (KGRC), Währinger Straße, 25, 1090, Vienna, Austria. E-mail: neil.barton@univie.ac.at.

${ }^{\dagger}$ Kurt Gödel Research Center for Mathematical Logic (KGRC), Währinger Straße, 25, 1090, Vienna, Austria. E-mail: sdf@logic.univie.ac.at.

¥The authors wish to thank Andrew Brooke-Taylor, David Corfield, Patrik Eklund, Michael Ernst, Vera Flocke, Deborah Kant, Cory Knapp, Colin McLarty, Chris Scambler, Georg Schiemer, Michael Shulman, Thomas Streicher, Dimitris Tsementzis, Steve Vickers, John Wigglesworth and [...] for helpful discussion and comments, as well as the editors for putting together the volume. They are also very grateful for the generous support of the FWF (Austrian Science Fund) through Project P 28420 (The Hyperuniverse Programme).

${ }^{1}$ This term is slightly coarse since it is ambiguous between material and categorial set theories (we will distinguish these later). For those that know the difference between the two types of set theory, we mean "material set theory" by "set theory" until we make the distinction, and lump categorial set theories in with category-theoretic foundations for now.
} 
One particular application to which category theory has been seen as especially suited to is elucidating the notion of mathematical structure $2^{2}$ A definition of mathematical structure is somewhat difficult to provide, but for the purposes of this paper we will take it that the existence of an isomorphism is sufficient for sameness of mathematical structure, and that this provides a useful way of getting at the notion (even if only partially).

This paper is directed at the following question:

Main Question. To what extent is (material) set theory a useful tool for discovering interesting facts about structures?

We will argue that set-theoretic language can be useful for conveying important structural information. In particular, we provide a theory of sets and classes which better respects isomorphism invariance, but nonetheless makes extensive use of the ambient material set theory. This is especially important if one holds that cardinality is a structural property; the theory we provide allows us to see how theories and cardinality interact (via a version of the Morley Categoricity Theorem).

Our strategy is as follows. First ( $\$ 1)$ we briefly outline the set-theoretic and categorial approaches, and explain the tasks to which each is best suited. Next (§2) we examine the difficulties that some proponents of each foundation have seen for the other party, and provide some responses on behalf of each. We argue that they are not in themselves problematic, but raise a challenge for the advocate of set-theoretic foundations. We then $(\S 3)$ present a theory of sets, urelements, and the classes that can be built over them. We argue that this language provides a modification of (material) set theory that better respects structural properties, whilst providing us with the resources to easily talk about notions like cardinality and how it interacts with structural notions. Finally $(\S 4)$ we make some concluding remarks and present some open questions.

\section{Two perspectives on foundations: Set-theoretic and Categorial}

In this section we'll explain the basic difference we see between category-theoretic and (material) set-theoretic foundations. We'll then refine both our set-theoretic and category-theoretic perspectives to give a better account of the subject matter they concern.

The distinction between the set-theoretic and category-theoretic perspective may be cast in different ways, however the most basic contrast is in how they approach the representation of garden-variety mathematical entities. The question is one of a perspective on which mathematics is about objects (and the internal membershipstructure those objects exhibit), versus one on which mathematics is about particular kinds of roles a mathematical object can perform within a wider context. Under settheoretic foundations, we focus on translating the language of a particular mathematical theory $\mathbf{T}$ into the language of set theory $\mathscr{L}_{\in}$ (consisting of only the language of first-order logic and a single non-logical symbol $\in$ ), and then find a model for $\mathbf{T}$ in the sets (given, of course, some antecedently accepted set theory). From the category-theoretic perspective, we would rather see what the essential relationships T-objects have to one another, and then try and capture these relationships through

\footnotetext{
${ }^{2}$ See, for example, [Awodey, 1996].
} 
the notions of arrow and composition. To see this difference, a couple of examples are pertinent:

Example 1. Singletons. In set theory the singleton of an object $x$ is the oneelement set $\{x\}$. Different singletons can look very different from the set-theoretic perspective; for example $\{\emptyset\}$ and $\left\{\beth_{\omega}\right\}$ are both singletons, but have very different properties (for example, their transitive closures look very different).

Conversely in category theory, we have the notion of a terminal object 1 , where an object 1 is terminal in a category $\mathcal{C}$ iff there is one and only one arrow from any other $\mathcal{C}$-object to 1 . Terminal objects can have a variety of different properties in different situations. For example, if we think of a partial order $\mathbb{P}=\left(P, \leq_{\mathbb{P}}\right)$ as a kind of category (so there is an arrow from $p_{0}$ to $p_{1}$ just in case $\left.p_{0} \leq \mathbb{P} p_{1}\right)$, then if $\mathbb{P}$ has a maximal element it will be terminal. Interestingly, in the set-theoretic context, we can form a category Set consisting of all sets as objects and the functions between them as arrows. We then see that between any set $A$ and any singleton $\{x\}$ there is exactly one function given by the rule $f(a)=x$ for every $a \in A$, and so the terminal objects of Set are exactly the singletons. Nonetheless, from the category-theoretic perspective it doesn't really matter which terminal object we talk about, since all terminal objects are isomorphic within any particular category. This contrasts sharply with the set-theoretic case where different singletons can have different interesting set-theoretic properties (such as identity of transitive closure).

Example 2. Products. In set theory, we define the product $A \times B$ of two sets $A$ and $B$ by first picking a canonical choice of ordered pair, and then letting $A \times B=\{\langle a, b\rangle \mid a \in A \wedge b \in B\}$ (giving us the 'rectangle' of $A$ and $B$ ). Products of more complicated objects are then defined component-wise. For example, the direct product of two groups $G=\left(D_{G}, *_{G}\right)$ and $H=\left(D_{H}, *_{H}\right)$ is defined as the following group:

$$
G \times_{\text {Group }} H=\left(D_{G} \times D_{H}, *_{G \times H}\right)
$$

Where $*_{G \times H}$ is defined component-wise for $g \in G$ and $h \in H$ :

$$
\left\langle g_{1}, h_{1}\right\rangle *_{G \times H}\left\langle g_{2}, h_{2}\right\rangle=_{d f}\left\langle g_{1} *_{G} g_{2}, h_{1} *_{H} h_{2}\right\rangle
$$

Conversely, in category theory, a product of two $\mathcal{C}$-objects $A$ and $B$ is another $\mathcal{C}$-object $A \times B$ together with two $\mathcal{C}$-arrows $\operatorname{Pr}_{A}: A \times B \rightarrow A$ and $\operatorname{Pr}_{B}: A \times B \rightarrow B$, such that for any pair of arrows $f: C \rightarrow A$ and $g: C \rightarrow B$, there is exactly one arrow $\langle f, g\rangle: C \rightarrow A \times B$ making the following diagram commute:

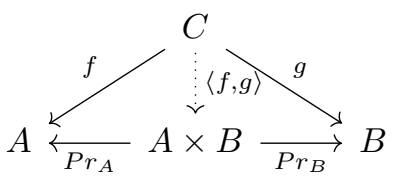


From the category-theoretic perspective, any objects that fulfil this role are a product (and, indeed, products are unique up to $\mathcal{C}$-isomorphism). In the case of sets and groups, the definition of set-theoretic product will (in Set) fulfil this role (using the relevant projections as $P r_{A}$ and $P r_{B}$ ), as will the definition of direct product for groups (when we consider the category Grp consisting of all groups as objects and group homomorphisms as arrows). However, any other definition of product resulting from a different definition of ordered pair would equally well qualify as a category-theoretic product (and indeed, we could find a function between the sets involved in the two products, 'factoring' one product through the other).

The difference in the above cases is the following: In set-theoretic foundations, representations of mathematical objects are obtained by construction from the membership relation and a suitable coding. On the category-theoretic perspective, we simply state what arrow-theoretic properties an entity must have in order to fulfil the functions it does in normal mathematical reasoning.

The eagle-eyed and/or well-informed reader may regard the distinction between set-theoretic and category-theoretic as a false dichotomy, since one can give categorial theories of sets by axiomatising the external functional properties attaching to the objects in a (or maybe 'the') universe of sets. This is precisely what is done on many categoria ${ }^{3}$ set theories such as on Lawvere's ${ }^{4}$ Elementary Theory of the Category of Sets (ETCS), which we'll examine in a little more detail later. In this way, it seems like the term 'set theory' can be correctly applied to certain categorial theories. For this reason we make the following distinction:

Definition 3. (Informal.) Material set theories are those that axiomatise a primitive notion of membership (denoted by ' $\in$ '), from which mathematical objects may be coded. Categorial set theory on the other hand provides a theory of sets formulated in the language of category theory, and on which objects are coded by systems of arrows resembling the usual properties we expect set-theoretic functions to have. Membership in categorial set theory is a defined relation, ofter ${ }^{5}$ explained in terms of functions $x: 1 \rightarrow A$ (read: " $x$ is a member of $A^{\prime \prime}$ ), since one can think of any such function (from a 'singleton') as 'picking' a member of $A]^{6}$

To make our initial question more precise, we are interested in the extent to which material set theory tell us interesting information about structures. Where the term "set theory" occurs without a qualifier, we mean material set theory and take categorial set theory to be a part of categorial foundations.

Both set theory and category theory allow us to identify objects up to structural equivalence. Exactly how they do so is a tricky issue, and provides us with a third:

Example 4. Isomorphisms. In set theory, working within first-order logic, we settle upon some relevant coding of vocabulary (i.e. function, constant, and relation

\footnotetext{
${ }^{3}$ There is some dispute over the use of the term 'categorial' versus 'structural' when axiomatising sets in category theory. We use the term 'categorial' since we reserve structure-like terms for the philosophical notion of structure.

${ }^{4}$ See [Lawvere, 1965] for the original presentation, and [Lawvere and McLarty, 2005] for an updated version. A clean and concise presentation (with some informal commentary) is available in [Leinster, 2014].

${ }^{5}$ As with many notions in category theory, there are different arrow-theoretic ways of getting at the same idea. See, for example, [Goldblatt, 1984] (Ch. 4) for some discussion.

${ }^{6}$ We are grateful to Michael Shulman and Dimitris Tsementzis for emphasising the importance of making this distinction.
} 
symbols), of structure (usually as an ordered tuple), and satisfaction on a structure of formulas in this language (given by an interpretation function on a structure). We then say that two structures in the same vocabulary $\mathfrak{A}$ and $\mathfrak{B}$ are isomorphic iff there is a (coded) bijection between their domains such that for every relation symbol $R$ of the vocabulary (respectively for function and constant symbols) and for every finite sequence $\vec{a}$ from $A, R^{\mathfrak{A}}(\vec{a})$ iff $R^{\mathfrak{B}}(f(\vec{a}))$.

Importantly (an issue often glossed over in mathematics) ${ }^{a}$ discussion of isomorphism only makes sense once the vocabulary (and some suitable coding thereof) has been chosen (on top of the coding-dependence of the set-theoretic analysis of bijection).

In category theory, however, the notion of isomorphism is dealt with by external arrow-theoretic properties. An arrow $f: X \rightarrow Y$ is an isomorphism (in a category $\mathcal{C}$ ) iff there is a $\mathcal{C}$-arrow $g: Y \rightarrow X$ in such that $g \circ f=I d_{X}$ and $f \circ g=I d_{Y}$ (i.e. composing the functions in either direction yields the identity morphism). Two objects are said to be isomorphic (in $\mathcal{C}$ ) iff there is an isomorphism between them. Importantly, the notion of isomorphism only makes sense within a category.

The treatment of isomorphism through a particular kind of arrow results in contexts in which the notion of set-theoretic and category-theoretic isomorphism come apart. For example, there are cases where we have category-theoretic isomorphisms that are not bijective homomorphisms (in the material set-theoretic sense). One such kind is when the relevant arrows are simply not functions, such as in the category of proofs which has sentences as objects and equivalence classes of proofs as arrows (so there is a single arrow $f: P \rightarrow Q$ when $Q$ is derivable from $P$ ). Here, since isomorphisms are equivalence classes of proofs between equivalent sentences, we have isomorphisms that are not (properly speaking) bijections of any kind. In the context where there is a functorial relationship between the category and Set, however, there can be no non-bijective isomorphisms (since functors preserve isomorphisms).

However, in the case where there is no functor between the category and Set, this is possible. An interesting (yet complex) case is the homotopy category that has topological spaces as objects and homotopy classes of continuous functions as arrows. Here, the inclusion of the unit circle into the punctured plane is an isomorphism (its inverse is the radial projection map), which is not bijective. In fact, [Freyd, 1970] showed that this is not a concrete category (i.e. there is no nice faithful functor from this category to Set), which facilitates the consideration of non-bijective iso-arrows $b_{c}^{b c}$

\footnotetext{
${ }^{a}$ See [Baldwin, 2018] (Ch. 1, esp. §1.2) for an argument that this is an often ignored distinction.

${ }^{b} \mathrm{I}$ am grateful to Andrew Brooke-Taylor for bringing this example to my attention, and discussion of the issue.

${ }^{c}$ A further simple (but somewhat silly) example is the following category which we define material-set-theoretically. The category has just one object $\{a, b\}$, and a single morphism defined by $f(a)=f(b)=b$. Here $f=I d_{\{a, b\}}$ (in the category), and so is trivially iso, but is nonetheless non-bijective.
}

The above example is important, since it shows that even the notion of structural similarity (as captured by the notion of isomorphism) is differently interpreted by the two perspectives. Thus, whether or not a property is 'isomorphism' invariant depends already on whether one holds one of the two perspectives to be privileged.

It should also be noted that talk of 'objects' is dispensable from the categorytheoretic perspective. Really, by taking the notion of domain and co-domain as part-and-parcel of the arrow, we could just speak purely in terms of arrows and 
composition. Material set theory and category theory thus represent two different perspectives on the nature of mathematical subject matter; on the one hand, we might think of mathematical objects as constructed and determined by their internal membership-structure, and on the other we might think of them as determined (up to isomorphism) by their relationships to other entities, and the role they play in the category as a whole.

This underlying difference in perspective represents two sides of a long-standing $9^{7}$ philosophical divide: Should we think of the subject matter of mathematics as given by individual objects with particular intrinsic relations determining their properties, or should we rather think of the subject matter of mathematics as concerned with purely structural properties (i.e. those invariant up to isomorphism)? The material set-theoretic and categorial perspectives are interesting representatives of different sides of this divide (though, as we shall see, issues are more subtle than they first appear) ${ }^{8}$

\section{Objections: Refining the perspectives}

As it stands, however, there are puzzles for each conception of foundations. In this section, we explain some of the complaints that have been made about the different foundational viewpoints and argue that these are easily resolvable. We'll argue that this suggests a possible route of inquiry for the friend of set-theoretic foundations; to modify their language in order to better respect isomorphism invariance and structure.

\subsection{Objections to categorial foundations and schematic types}

One supposed 'problem' for the friend of category-theoretic foundations concerns its subject matter. What, exactly, is category theory about? For, as it stands, category theory merely defines particular kinds of algebraic structure. The discipline seems to be of a piece with algebraic enterprises such as group theory or other areas of abstract algebra. One begins by laying down conditions on what a system of arrows must satisfy in order to be a category (existence of identity and composition morphisms, and associativity of composition). This defines an algebraic structure much like that of group (in fact, there is a corresponding abstract algebraic structure for category that is slightly more general than that of group; namely then notion of being a monoid), which can then be made more specific with additional constraints. For example, insisting that particular diagrams exist and commute in a category yields the definition of a topos: a Cartesian closed category with a subobject classifier. This kind of category is very useful for studying the algebraic properties instantiated by various logical and mathematical systems, and while it is exceptionally rich in structure, it still (in the spirit of category theory) just corresponds to particular algebraic properties that a system of arrows can instantiate. Hellman sums up this thought:

"...this theory [i.e. category theory] itself is presented algebraically, via first-order 'axioms' only in the sense of defining conditions telling us what a category is, together with further ones defining topoi of various sorts.

\footnotetext{
${ }^{7}$ At least since [Benacerraf, 1965].

${ }^{8} \mathrm{~A}$ salient third option (especially given the topic of the current volume) is Homotopy Type Theory. Here type theory endowed with a homotopy-theoretic interpretation is employed, providing a foundation that meshes elegantly with category-theoretic methods. See the excellent |The Univalent Foundations Program, 2013| for discussion.
} 
As such these 'axioms' are like the conditions defining a group, a ring, a module, a field, etc. By themselves they assert nothing. They merely tell us what it is for something to be a structure of a certain kind." ([Hellman, 2006], p. 134)

Hellman's point (an important one) is that the axioms of category theory (even when expanded to isolate categories of more complex varieties) make no existential claims (this is what Hellman means by saying that the axioms "assert nothing") in that they specify what it is for a system of objects to satisfy certain axioms, without asserting that anything satisfying them actually exists.

Some (including [Hellman, 2006]) have taken this as an objection to categorytheoretic foundations. An appropriate foundation for mathematics should state that some objects exist and that mathematics can be interpreted within this structure, thereby laying a framework ontology on which mathematics can be built. Thus category theory appears to contrast with the usual set-theoretic foundations, where the axioms of Infinity, Power Set, and Replacement all make existential claims within ZFC, and many other axioms extending ZFC also make existential claims ${ }^{9}$

This objection should not trouble the friend of category-theoretic foundations. One salient response (made by [Mclarty, 2004]) is that no-one has ever proposed the axioms of category theory as a foundation, the proposal is rather to assert that some topos or other exists and mathematics either can or should be interpreted there. Good examples here are categorial theories of sets (such as ETCS) or attempts to axiomatise a category of all categories (e.g. CCAF).

Moreover, we might also think that Hellman's objection simply misses the mark. His remarks reveal something about the general practice of category theory: It is an algebraic discipline, no matter whether it can be modified to yield assertory content, as McLarty suggests. When practising category theory, we care only about whether we have the relevant relations between objects, and this does contrast with set theory where (largely speaking) we are interested in the properties of some specific structure (namely the cumulative hierarchy) ${ }^{10}$ This feature of the two frameworks is further witnessed by attitudes to categoricity. In a categoricity proof, we aim to show that a certain theory has just one model (up to isomorphism). In the context of set theory (or indeed number theory and analysis) the project of providing a categoricity proof makes sense; we wish to see that our axiomatisation has been successful and we have (given the determinacy of the concept) pinned down a single structure up to isomorphism ${ }^{11}$ In the case of number theory and analysis we have proofs of full categoricity (by the work of Dedekind), and in the case of set theory we have quasi-categoricity: We can show that $\mathbf{Z F C}_{2}$ augmented with anti-large cardinal axioms can be fully categorical, and any two $\mathbf{Z F C} \mathbf{C}_{2}$ structures are either isomorphic or one is isomorphic to an initial segment of the other ${ }^{12}$ In the case of category theory though, to attempt such a proof for a categorial theory would be an absurd endeavour, the whole point of category theory is to isolate structural properties that can be

\footnotetext{
${ }^{9}$ Good examples here are so called large cardinal axioms, as well as forcing axioms, and inner model hypotheses.

${ }^{10}$ Here we are playing slightly fast-and-loose with debates in the foundations of set theory; under a natural interpretation of Joel Hamkins' multiverse perspective, set theory also should be understood as purely algebraic. See [Hamkins, 2012] for the original presentation of this view and [Barton, 2016] for an argument to the effect that this results in a purely algebraic interpretation.

${ }^{11}$ The exact dialectic import of a categoricity proof is something of a vexed question, see [Meadows, 2013] for discussion. An argument that the quasi-categoricity of $\mathbf{Z F C}_{2}$ shows that our axiomatisation has been successful is available in [Isaacson, 2011].

${ }^{12}$ The original proof of this is available in [Zermelo, 1930], and is subsequently tidied up in [Shepherdson, 1951] and [Shepherdson, 1952].
} 
shared by radically non-isomorphic structures.

Thus categories demand no single place to be interpreted, and the complaint that category theory fails to delimit a determinate range of objects is misplaced. The friend of set-theoretic foundations, for example, will regard it as of a piece with group theory; if category-theory has any subject matter at all, it is the categorytheoretic structure that can be instantiated in various ways in the sets (and so she should countenance category theory as a useful foundational language, even if it is not her favourite foundation). There is no pressure to find 'the' structure of 'the' category-theoretic world; the discipline is designed to be flexible and resist such a characterisation.

A friend of category-theoretic foundations might thus regard the subject matter of mathematics as fundamentally algebraic, category theory as providing a good axiomatisation of this perspective, but nonetheless resisting the characterisation of a unique concrete subject matter. If category theory has a subject matter at all, then it is a purely structural one.

An immediate and difficult question is how we should think of this categorytheoretic structural subject matter. Landry and Marquis provide the following interesting idea:

"A category, too, is neither a privileged abstract kind of system nor is it an abstract Fregean "structure" qua an "object": it is a Hilbertian style abstract structure qua a schematic type, to be used as a framework for expressing what we can say about the shared structure of the various abstract kinds of structured systems in terms of 'having' the same type of structure." ([Landry and Marquis, 2005], p. 35)

The thought here is to think of categories as providing structure as a 'schematic type', rather than a 'particular structure'. Of course, it bears explaining what a 'schematic type' is. One kind of schematic type is well known to mathematical logicians - the notion of first-order theory. These, if they can be instantiated in any infinite context, can be instantiated in many infinite contexts. We leave it open whether non-first-orderisable content can be expressed categorially. If higher-order content (with a version of the full semantics) can be encoded in a categorial language, then it is at least possible that we might determine a structure up to (set-theoretic) isomorphism categorially. Whether or not this is possible we leave open; for now, we note that the ability to systematise relationships across non-isomorphic contexts just is one of the main strengths of category theory, and many proponents of categorial foundations do see category theory in this light (e.g. [Mac Lane, 1986]).

Thus we take the target of category theory to be showing the basic relationships objects have to have to one another to fulfil their functional roles. In order to understand better the notion of schematic type, it is useful to return to the analogy with group theory. While it makes sense to speak of 'the group-theoretic structure', there is not a single way the group-theoretic structure can be instantiated, rather it admits of satisfaction in multiple different ways and contexts (and indeed this is one of the reasons why abstract algebra has been so fruitful in contemporary mathematics). This is much the same for categories, which provide a useful framework for systematising these relationships. We thus provide the following:

Definition 5. (Informal and philosophical) A schematic type is a system of relationships that can be instantiated in many different non-isomorphic contexts.

Viewing category theory as the appropriate theory for formalising schematic types, we see that the problem of 'subject matter' is not really a problem at all. 
Rather, category-theoretic foundations provide a language and context in which to study algebraic relationships, and for this reason precisely resist the identification of a concrete subject matter. Categories correspond to theories of mappings and can be multiply instantiated throughout concrete systems of objects, and there is no pressure to identify a unique subject matter or make it assertory in nature ${ }^{13}$

\subsection{Objections to set theory and the combinatorial perspective}

In this subsection, we'll delve into some of the criticisms of material set theory. We'll show that when set theory is understood as applying to an appropriate subject matter (namely providing an analysis of possible combinations of objects) the objections fail to gain traction. We'll therefore suggest that a methodological pluralism is an attractive attitude to foundations. Before moving onto the final section, we'll identify that a possible line of inquiry for the friend of set-theoretic foundations is to provide a modification of her language that better respects isomorphism invariance.

The objections to set-theoretic foundations come in two broad kinds, as Feferman (speaking about [Mac Lane, 1971]) explains:

"Two views are intermixed in [Mac Lane, 1971] as to current set-theoretical foundations, namely that (i) they are inappropriate for mathematics as practised, and (ii) they are inadequate for the full needs of category theory." ([Feferman, 1977], p. 149)

Our strategy will be the following. We expand on Feferman's two dimensions, articulating the different objections we find in the literature. For reasons that will become apparent (we find the former dimension to be the more challenging of the two), we deal with these in reverse order, starting with inadequacy. As we go, we will provide responses from the set-theoretic standpoint. We do, however, have to be careful about dialectical strategy. In 'defending the set-theoretic viewpoint' we could be doing one of (at least) two things:

1. We could be arguing that, despite the category-theoreticians best efforts, set theory is still the best foundation for discussing mathematical structure.

2. Slightly weaker, we could contend that despite arguments to the contrary, set theory is still interesting as a foundation as it has plenty to tell us about mathematical structure.

We wish to emphasise that it is this latter claim we wish to support. We wish to claim that despite many criticisms in the literature, set theory can still provide interesting information about how certain structural properties are instantiated.

\subsubsection{Inadequacy}

The problem of inadequacy is roughly the following: Set theory does not provide enough of something (either objects or information). The key issue is raised by Mac Lane:

\footnotetext{
${ }^{13} \mathrm{~A}$ second objection, one that we will not consider here, is the point raised by [Mathias, 2000] and [Mathias, 2001] that category theory lacks the logical strength to discuss certain strong statements of analysis that relate to large cardinal axioms. While the objection merits a response, we set this aside for several reasons: (1) research is ongoing here, and it is unclear that category theory cannot do the job, (2) there are, in any case, logically strong category-theoretic statements (see below), and (3) the possible responses to the objection do not help us elucidate the philosophical role being played by category theory in terms of schematic types.
} 
"Our fundamental observation is just this: There is an appreciable body of results about categories...but the received methods of defining categories in terms of sets do not provide any one single context (i.e. any one model of a standard set theory) within which one can comprehensively state these results." ([Mac Lane, 1971], p. 235)

The fundamental idea is the following: It is undeniable that the methods of category theory have provided a versatile method for modern mathematics. This raises the question for the friend of set-theoretic foundations: "Given that category theory provides structural information, what sets should we interpret category theory as about?".

Mac Lane's point is that there is no single context in which we can interpret category theory unrestrictedly. This is visible in two related but distinct dimensions: (i) Which mode ${ }^{114}$ we should take to found category theory, and (ii) Which categories we should expect set theory to found ${ }^{15}$

What model? The first issue concerns exactly what the relevant model should satisfy. Simply put, the widespread independence phenomenon in set theory has challenged the classical idea that there is a single maximal universe of sets in which we may interpret all mathematical discourse. This is discussed by Mac Lane:

"These results, and others too numerous to mention, show that many interesting Mathematical questions cannot be settled on the basis of the Zermelo-Fraenkel axioms for set theory. Various additional axioms have been proposed, including axioms which insure the existence of some very large cardinal numbers and an axiom of determinacy (for certain games) which in its full form contradicts the axiom of choice. This variety and the undecideability results indicate that set theory is indeterminate in principle: There is no unique and definitive list of axioms for sets; the intuitive idea of a set as a collection can lead to wildly different and mutually inconsistent formulations. On the elementary level, there are options such as ZFC, ZC, ZBQC or intuitionistic set theory; on the higher level, the method of forcing provides many alternative models with divergent properties. The platonic notion that there is somewhere the ideal realm of sets, not yet fully described, is a glorious illusion." ([Mac Lane, 1986], p. 385$)^{16}$

Since our expertise is primarily in higher-set theory (i.e. ZFC and its extensions) and independence, we approach the issue from that perspective. As is well-known, there are many set-theoretic sentences independent of our canonical set theory ZFC (e.g. $\mathrm{CH}$ ). Mac Lane takes this to show that there is no one notion of 'set' and hence no one place that category theory can be interpreted. We have two responses to this argument:

Response 1. This explicitly takes a stand on the status of certain questions in the philosophy of set theory. In particular, it turns on how many universes of set there are (or at least how many distinct but legitimate concepts of set there are). While the

\footnotetext{
${ }^{14}$ We use the term 'model' in a loose and informal way here, and intend it to apply to possibly properclass-sized structures. For example, we will at least allow $(L, \in)$ as a model, even though it is proper-classsized.

${ }^{15}$ In the quotation above, Mac Lane is specifically interested in the first point we consider. However, the intuition expressed transfers naturally to other objections he makes, as outlined below.

${ }^{16}$ Similar remarks are made repeatedly in [Mac Lane, 1986], cf. pp. 359-360, 373.
} 
independence phenomenon is certainly challenging, this does not mean that there are multiple 'meanings' to the word "set". Thus, for the theorist who simply rejects the claim that the independence phenomenon indicates semantic or ontological indeterminacy, the objection gains no traction (without further argument).

Response 2. Even if we allow the existence of different universes or concepts of set, Mac Lane's criticism is subject to a tu quoque. This is because, as we explained above, category theory by its nature does not demand a single context for interpretation (in fact quite the reverse). Rather, we argued, category theory should be understood as providing a uniform language in which to study algebraic properties and schematic types. Thus to insist on a single model or axiomatisation in which category theory should be interpreted is to impute content to it that is simply not there. Thus, insofar as this is a problem for set theory at all, it is also one for categorial foundations.

This allows a quick response to Mac Lane's objection: Even if there are multiple set-theoretic concepts or universes, and no overarching context, this does not matter. Wherever we study category-theoretic properties set-theoretically (discussion of particular set-theoretic interpretations is provided below), we know that our results will transfer to the alternative cases via the schematic properties of category theory. It is enough for us to study category-theoretic structure set-theoretically to find one set-theoretic structure exemplifying the relevant schematic type. For the purposes of set-theoretic foundations, we do not need to find the set-theoretic subject matter corresponding to category theory ${ }^{17}$

Which categories? The second problem of inadequacy concerns what one has within a particular context. Simply put, category theory seems to speak about structures that are proper-class-sized, and so do not have any set-theoretic representative. An obvious example here is Set, the category of all sets that has as arrows set-theoretic functions (this can be given direct category-theoretic axiomatisation by ETCS or its extensions) 18 There are, however, many such categories (e.g Grp, Top, Fld, etc.).

There are two main strategies for overcoming this problem. The first is to posit the existence of Grothendieck universes and interpret category theory there. More formally:

Definition 6. A Grothendieck universe is a set $U$ with the following properties:

(i) $U$ contains the set of all natural numbers.

(ii) $U$ is transitive.

(iii) $U$ is closed under the formation of pair sets.

(iv) $U$ is closed under the formation of power sets.

(v) $U$ is closed under set-theoretic union.

(vi) If $x \in U, f: x \rightarrow y$ is a surjection, and $y \subseteq U$, then $y \in U$.

We can then interpret category theory as concerned with any such universe. For instance, Set can be interpreted as concerned with the sets in some $U$ (let's call this

\footnotetext{
${ }^{17} \mathrm{~A}$ similar point is made in [Maddy, 2017]. For some other remarks on what we would like from settheoretic foundations, see also Maddy's contribution to the present volume [Maddy, F].

${ }^{18}$ The topos axiomatised by ETCS is that of a well-pointed topos with a natural number object and satisfying the categorial version of the Axiom of Choice.
} 
category $\operatorname{Set}_{U}$ ), and this (along with any functor categories) is a perfectly legitimate object formed in the stages of the cumulative hierarchy above $U$.

Grothendieck himself (in proposing a set-theoretic interpretation of category theory) suggested the axiom that there should be an unbounded sequence of these universes. In fact, being a universe is clearly equivalent to being $V_{\kappa}$ where $\kappa$ is an inaccessible cardinal, and so the proposal comes down to interpreting category theory within any one of an unbounded sequence of inaccessible universes.

The standard objection to this strategy is that it apparently 'prevents' considering perfectly acceptable categories, such as the actual category of all sets. Given any such interpretation of category theory, there are sets outside of that interpretation. But (so the objection goes) category theory is about any and all sets instantiating the relevant category-theoretic interpretation. Any such restriction seems ad hoc. Muller expresses the point as follows:

"Any stipulation to the effect that the category-theoretician is only allowed to grab at some fixed set whereas outside this set there are more sets, so that he is not permitted to grab at all of them, is artificial and barks at his explicit intentions. The category- theoretician has every right to refuse to dance to the cardinality tunes [of] the set-theoretician whistles. Category-theory is about form \& structure, irrespective of how much \& how many; it 'only' wants to have everything which is available. The category-theoretician means all sets when he makes the category Set of all sets, period. Set-theories which cannot accommodate this are flawed." (Muller, 2001], p. 11-12)

A different strategy then is to allow proper-class-sized categories (so-called 'large' categories), and adopt a two-sorted class-theoretic language and theory (such as NBG or MK) in providing a category-theoretic interpretation. The problem here is that often category-theorists will consider functor categories between two categories. Taking two categories $\mathcal{C}$ and $\mathcal{D}$, it is natural to consider the category $\mathcal{D}^{\mathcal{C}}$ consisting of all functors from $\mathcal{C}$ to $\mathcal{D}$ as objects, and natural transformations between functors as arrows. Such a category, however, is often one type higher than both $\mathcal{C}$ and $\mathcal{D}$. In the case then when both $\mathcal{C}$ and $\mathcal{D}$ are large, even with proper classes we are not guaranteed the existence of a class-theoretic representative concerning $\mathcal{C}^{\mathcal{D}}$ (normally proper classes cannot be members) 19 The issues concerning both interpretations are summed up as follows:

"Using universes, all the functor categories are there, but there is no category of all groups. Using Godel-Bernays, one has the category of all (small) groups, but only a few functor categories.'20 ([Mac Lane, 1971], p. 235)

What should we take from this? Again we hold that these objections fail to gain traction:

Response 1. A simple point, but one based on a non-trivial theorem, is that for certain categories (in particular the category of all reflexive graphs), it is not clear

\footnotetext{
${ }^{19}$ Of course, the material set-theorist might just accept the existence of proper-classes, hyper-classes, hyper-hyper-classes and so on. This is naturally interpretable in an ontology on which every universe can be extended in height, but there is also a question of whether the believer in one maximal unique universe of sets could also make use of $n^{\text {th }}$-order hyper-classes. Normally it is assumed not, but this question remains philosophically open.

${ }^{20}$ Given the topic of the present volume, there is an interesting question as to the extent to which this difficulty is avoided in homotopy type theory. We thank Dimitris Tsementzis for the suggestion that this difficulty could possibly be overcome in this foundation.
} 
that the requirements Mac Lane wishes to place on interpretations of category theory are consistent. Specifically [Ernst, 2015] shows that there are restrictions on what categories one can have. The proof proceeds by considering a version of Cantor's Theorem in the category of all reflexive graphs and shows that certain desirable conditions on the existence of categories are jointly inconsistent ${ }^{21}$ Thus we cannot simply naively insist on the existence of any category whatsoever without some restrictions.

Response 2. The problem implicitly takes a stand on issues in the philosophy of set theory, though in the opposite direction to the previous section. For, in the case where we think there is no maximal conception of the notion of ordinal, and rather that any universe of set theory can be extended in height, we are always implicitly restricted to a particular bounded universe of sets anyway. Thus, to the theorist who holds that there are no 'maximally high' universes, the objection fails to gain any traction.

Response 3. Even if we do allow that there can be 'maximally high' universes of set theory, the objection again fails to account for the schematic nature of category theory. Given this algebraic character, when a category theorist asks us to consider "The category Set which has as objects all sets and arrows all functions" this should be understood as shorthand for communicating various category-theoretic properties. Some of these are captured by first-order axiomatisations such as ETCS and its extensions, but again, they are about a schematic type (in particular a first-order one) rather than a particular concrete subject matter. So for studying this schematic type, it is enough that we have just one structure exemplifying the schematic type. Results proved about this schematic type can then be exported to other contexts (and other structures instantiating the category-theoretic properties), the results are about the schematic type and not the concrete instantiation of it in the sets.

Consider the case where we have both the Grothendieck-universe and classtheoretic interpretations available to the set theorist. More precisely, suppose she believes the following: (1.) There is a single unique universe of sets, (2.) There are unboundedly many inaccessible cardinals, and (3.) There is a satisfactory interpretation of Morse-Kelley class theory over $V$. Now, consider the category theorist's consideration of Set, and how this relates to the set theorist's universe. The categorytheoretic structure of Set is multiply instantiated, both by each individual $V_{\kappa}$ where $\kappa$ is inaccessible, and also $V$ (as well as many other structures besides, some of them countable ${ }^{22}$. Now suppose we consider some 'super-large' functor category $\mathbf{S e t}^{\mathcal{C}}$ (for some category $\mathcal{C}$ ). The $\operatorname{Set}^{\mathcal{C}}$ schematic type will be instantiated by the various $V_{\kappa}$ with the sets above them, but not by $V$ (since there are no levels above $V$ ). But this does not matter, any properties proved about the schematic type Set using the schematic type $\operatorname{Set}^{\mathcal{C}}$ can just be exported back to $V$, the proof concerning Set just depends on there being some set-theoretic counterpart in which $\operatorname{Set}^{\mathcal{C}}$ is instantiated, not that every instantiation of Set has a corresponding $\operatorname{Set}^{\mathcal{C}}$ representative. While it is the case that when a category theorist states a theorem of the form "Set has an extension to $\mathbf{S e t}^{\mathcal{C}}$ " can only be interpreted to concern the small instantiations, this does not harm the results about the schematic type Set, and the fact that any results proved on the basis of $\mathbf{S e t}^{\mathcal{C}}$ about that structure can perfectly well be exported back

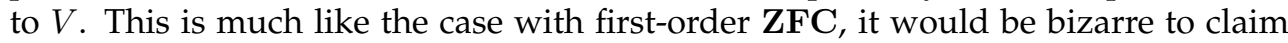
that results proved about ZFC using extensions in the model theory of sets are in-

\footnotetext{
${ }^{21}$ See [Ernst, 2017], |Maddy, 2017] and | Maddy, F| for further discussion of the significance of this result. ${ }^{22}$ An example of such a structure would be the Skolemisation and Mostowski Collapse of any settheoretic structure satisfying an appropriate amount of set theory.
} 
applicable to $V$ because $V$ lacks extensions ${ }^{23}$

A natural rejoinder is that occasionally category theorists will consider explicitly large categories like SET (i.e. the category of all sets) rather than just Set (interpreted as the category of small sets in the first universe). Nonetheless, exactly the same considerations apply concerning schematic types. Even if one insists that there is a distinction between 'small' and 'large' sets, one can still have SET instantiated in some small structure, it is just that that structure does not think it is small. These considerations are familiar from the set-theoretic framework; one can easily have a particular $V_{\kappa}$ satisfying the claim that there are proper-class-many cardinals of some kind $\Phi$, without $V_{\kappa}$ witnessing that there are literally (i.e. in $V$ ) proper-class-many such cardinals. One just requires that the $\Phi$-cardinals are unbounded in $V_{\kappa}$ for there to be a universe satisfying this property. Indeed, as above, any conclusions based on the theory ZFC+ "There is a proper class of $\Phi$-cardinals", made by considering the extension of a model thereof can be exported back to $V$ (on the assumption of course that $V$ does contain such a proper class). Similarly, one just needs a universe containing an inaccessible (in fact if the schematic content of category theory is only first-order, one needs much less) for there to be set-sized set-theoretic contexts in which there is a meaningful distinction between small and large categories capable of instantiating the relevant algebraic content. To argue that set theory fails to provide an appropriate surrogate for $\mathbf{S E T}^{\mathcal{C}}$ is to impute non-algebraic content to category theory which is quite simply not there.

\subsubsection{Inappropriateness}

The inappropriateness dimension of Feferman's taxonomy concerns set theory giving us too much of something (either objects or information). We'll see that while these problems are also resolvable, an additional line of inquiry is suggested by the complaint that set theory provides too much non-isomorphism-invariant information; namely to modify set theory so that the language respects isomorphism invariance.

Logical strength. The first issue concerns the logical strength of set theory. For the practical purposes of founding mathematics, so the argument goes, we do not need anything like the strength of ZFC set theory. Landry and Marquis record this sentiment:

"Second, it is fair to say that category theorists and categorical logicians believe that mathematics does not require a unique, absolute, or definitive foundation and that, for most purposes, frameworks logically weaker than $\mathbf{Z F}$ are satisfactory. Categorical logic, for instance, is taken to provide the tools required to perform an analysis of the shared logical structure, in a categorical sense of that expression, involved in any mathematical discipline." ([Landry and Marquis, 2005], p. 19)

We do not wish to disagree that large portions of mathematics do not require the logical strength of ZFC set theory. However, we do wish to make two rejoinders:

Response 1. First, set theory does not aim at being 'minimal' in any sense. Rather, we wish to provide the most generous theory possible (often understood through

\footnotetext{
${ }^{23}$ There may, nonetheless, be certain philosophical considerations here, as well as technical issues concerning how much higher-order reasoning we can capture using extensions. See [Barton, S] and Antos et al., S] for discussion.
} 
maximising consistency strength) to be used in interpreting any conceivable mathematics we might consider. So, while the objection might be convincing to a theorist who has a penchant for minimising logical strength, it fails to be convincing to the friend of set-theoretic foundations.

Response 2. Second, we have another tu quoque here: There are interesting category-theoretic principles that turn our to have significant large cardinal strength. Bagaria and Brooke-Taylor, for example, note the following (in an article on colimits and elementary embeddings):

"Many problems in category theory, homological algebra, and homotopy theory have been shown to be set-theoretical, involving the existence of large cardinals. For example, the problem of the existence of rigid classes in categories such as graphs, or metric spaces or compact Hausdorff spaces with continuous maps, which was studied by the Prague school in the 1960's turned out to be equivalent to the large cardinal principle now known as Vopěnka's Principle... Another early example is John Isbell's 1960 result that Set ${ }^{\mathbf{O p}}$ is bounded if and only if there is no proper class of measurable cardinals." ([Bagaria and Brooke-Taylor, 2013], p. 1)

A key point to attend to in the above is that these are not category-theoretic principles that were dreamt up by set theorists. These are principles that were naturally studied by category theorists that turned out to not just be independent from ZFC, but also have substantial large cardinal strength (Vopĕnka's Principle is quite high in the large cardinal hierarchy). Moreover, with certain additional assumptions one can find models of material set theories like ZFC in categorial set theories like ETCS ${ }^{24}$ In virtue of this, the claim that set theory is somehow unfavourably distinguished by its logical strength when compared to category theory (in all its guises) seems dubious.

Isomorphism invariance. The second problem of inappropriateness concerns the earlier discussed fact that set theory makes decisions about non-isomorphism invariant facts. The key issue is that a central practice in set-theoretic foundations involves specific choices of 'canonical' representatives.

For example, in the earlier discussion of products, the exact object that is eventually selected as 'the' product will vary depending on numerous conventional stipulations. We might, for example, represent the ordered pair $\langle a, b\rangle$ along the lines of Hausdorff as $\{\{a, 1\},\{b, 2\}\}$ (rather than the usual Kuratowski definition:

$\left.\langle a, b\rangle=_{d f}\{\{a\},\{a, b\}\}\right)$ resulting in a different choice of product. This then raises the following question: If set theory is meant to tell us what mathematical objects exist, then what is the fact the matter about which definition of product is the actual product?

The problem was noticed in the philosophical literature at least as early as the seminal [Benacerraf, 1965]. There, he presses this problem of non-isomorphism invariant choices to be made via the existence of 'junk' theorems, where a theorem is 'junk' when it concerns non-isomorphism invariant properties. For example, is it true that $5 \in 7$ ? A natural answer is "Yes"; the canonical choice of ordinals is the von Neumann definition, and under that definition it is the case that $5 \in 7$. However, under the Zermelo definition, it is not the case that $5 \in 7$. So, if we think that

\footnotetext{
${ }^{24}$ For instance one way to do this is to find arrow-theoretic trees in a model of strengthened ETCS that correspond to the relevant membership trees required to build a model of ZFC. We are grateful to Michael Shulman for discussion here, and directing us to his useful [Shulman, 2010] and [Osius, 1974].
} 
mathematical objects 'are' sets (or, as Benacerraf points out, any objects) what is the fact of the matter concerning whether or not $5 \in 7$ ? Since the truths of number theory are invariant under domain (we just need some things that have the standard natural number structure under the relevant relations of the required vocabulary for arithmetic to be true in that context) there seems to be no good response; whatever objects we pick as 'the' natural numbers, they satisfy the same arithmetic sentences.

The simple response is that the many (if not most) set theorists do not subscribe to the heavy-duty set-theoretic reductionism required to generate the problem. Rather, most friends of set-theoretic foundations (or, at least, this is the line we shall present here), take set theory to be a device of representation. Using the membership relation and axioms of first-order logic we are able to interpret mathematical claims as ones about sets, with axioms telling us how these objects can be combined to yield other mathematical properties, providing a context in which different mathematical claims can be interrelated.

Claims like " $5 \in 7$ " just do not make sense for the friend of set-theoretic foundations until we have settled on a particular interpretation of number theory. If we pick the von Neumann ordinals, then the question of whether $5 \in 7$ corresponds via our chosen interpretation to whether $5<7$ in number-theoretic terms, and so is obviously true (in fact trivially so). If we had picked a different representation, however, it may have been false (as is the case on the Zermelian conception of finite ordinals).

This has implications for the purposes to which set theory is put in foundations. The language is excellent for studying what kinds of mathematical properties are compossible, and what kinds of objects are required to find an interpretation of a piece of mathematics. Hence, the default context for studying independence results (and their implications) is models of set theory ${ }^{25}$ and the indexing of consistency strength is accomplished using set-theoretic principles. If one wishes to know whether it is possible to have one mathematical property with or without another, one studies the models of set theory in which they have interpretations. If one wishes to know how much logical strength is implied by the adoption of a particular mathematical system, a standard technique is to find a model of the principle in the sets. However, once a model has been found, few set theorists (if any) would suggest that the mathematician should change their practice and adopt the (highly baroque) settheoretic language. The interpretation has been given, and this acts as a certificate that the original (and probably more fluid) language is in good working order ${ }^{26}$

This has implications for the kind of applications that we see for set theory to mathematical structure. Rather than seeing it as a language and framework for the working mathematician, it should rather be used in finding interpretations of the working language and comparing them. Structurally, this might have important consequences. One might, for example, see cardinality as an important structural property. Set theory then provides us with a useful framework in which schematic types of certain kinds and cardinality interact, to yield helpful structural informa-

\footnotetext{
${ }^{25}$ This said, there are category-theoretic options here. See [Bell, 2011].

${ }^{26}$ Vladimir Voevodky himself was clear about this role for ZFC with respect to Homotopy Type Theory. See, for example, his abstract for the 2013 North American Meeting of the Association of Symbolic Logic, where he says:

"Univalent foundations provide a new approach to the formal reasoning about mathematical objects. The languages which arise in this approach are much more convenient for doing serious mathematics than $\mathbf{Z F C}$ at the cost of being much more complex. In particular, the consistency issues for these languages are not intuitively clear. Thus ZFC retains its key role as the theory which is used to ensure that the more and more complex languages of the univalent approach are consistent." (|ASL, 2014], p. 108)
}

We are grateful to Penelope Maddy for bringing this to our attention. 
tion. A good example here is the celebrated:

Theorem 7. The Morley Categoricity Theorem. Suppose that a countable first-order theory $\mathbf{T}$ has exactly one model up to isomorphism in a single uncountable cardinal. Then it has one model (up to isomorphism) in every uncountable cardinal.

The standard proof of this proceeds against a background of material set theory. Assuming that one does hold that cardinality is a structural property, it yields information about how first-order schematic types and cardinality interact, specifically if one has a first-order schematic type $\mathbf{T}$ (this could even be given categorially) it provides conditions that tell us when there is only one way (up to isomorphism) that $\mathbf{T}$ could be instantiated in a structure of a particular size.

We thus make the preliminary conclusion; though material set theory is baroque and choices must be made about canonical representatives in a fashion that is not isomorphism invariant from the perspective of certain vocabularies (e.g. number theory), it nonetheless provides a useful perspective for stating how schematic types interact with other structural properties (namely cardinality). We thus subscribe to a methodological pluralism in foundations; category theory is the appropriate theory for explaining how schematic types interact with one another, but set theory is the appropriate language for explaining how schematic types interact with concrete systems of objects ${ }^{27}$

This response is satisfactory as far as it goes. However, a challenge remains for the set theorist: Could we possibly factor out the use of canonical representatives to yield a conception of set on which we are able to use the combinatorial power of set theory whilst considering isomorphism invariant properties?

The point is brought into especially clear focus when contrasting material set theory with categorial theories of sets (like ETCS). We should note that meaningful statements of categorial set theories like ETCS are not fully isomorphism invariant. Take, for example, the claim " $f$ has co-domain $B$ " for some $f$ and $B$. This interpretable as a perfectly good formula in the language of category theory, but is obviously not isomorphism invariant; there might be objects isomorphic to $B$ which differ as to whether they are the co-domain of $f$ or not ${ }^{28}$ However, something that ETCS does offer is a way of 'modding out' this non-isomorphism invariant noise. For instance, one can prove:

Theorem 8. (ETCS) Let $\phi(X)$ be a formula in the language of ETCS with no constants and no free variables except the set variable $X$. Then if $X$ and $Y$ are isomorphic, then $\phi(X)$ iff $\phi(Y) 2^{29}$

Thus ETCS provides us with a clean-cut class of formulas for which we have isomorphism invariance. Our task for the rest of this paper is to make a preliminary step in providing a material set theory that does the same for structure. We will

\footnotetext{
${ }^{27}$ The following analogy may be helpful. Viewing mathematics as describing a kind of quasicomputational enterprise, set theory is something like a theory of machine-code: It tells us what kinds of things can be built, and what we need to build them. Category theory on the other hand is like a highlevel programming language, codifying what effects can be achieved by different structural relationships in different contexts. Both the set theorist as computational engineer and the category theorist as programmer have important roles to play in mathematics. This analogy was originally communicated to the first-author by David Corfield after a talk at the LSE in November 2013. He is also grateful to Dr. Corfield for subsequent discussion of categorial foundations.

${ }^{28}$ There are some theories that aim to make it impossible to state non-isomorphism-invariant properties in their language. Two candidates here are Makkai's FOLDS (see [Makkai, 1998], [Marquis, 2017]) and Univalent Foundations (see |The Univalent Foundations Program, 2013|, |Tsementzis, 2016|). We are grateful to Dr. Tsementzis for pressing us on this point.

${ }^{29}$ See here [McLarty, 1993], p. 495.
} 
do so by proposing a theory of sets and classes built over structures composed of urelements, and end up with a theory that better respects isomorphsim invariance whilst facilitating the consideration of how schematic types and systems of concrete objects interact (as in the Morley Categoricity Theorem).

\section{How set theory helps the structural perspective}

We now have a challenge for the friend of set-theoretic foundations; find a use of settheoretic language that better respects isomorphism invariance. In this section, we do just that. The broad idea is to think of material set theory as built over some mathematical subject matter, conceived of as composed of structures with urelements and the functions and relations between them. Our point is the following; we can factor out the arbitrary choices of coding to find a material set theory that by design respects isomorphism invariance.

\subsection{Set Theory with Structures (ZFCS)}

In taking inspiration from structuralism 30 we will consider structures as composed of featureless points, and the functions and relations between them. Treating a featureless point as a kind of urelement, we will build a theory of sets and classes over these urelements, and show how by doing so we can develop a more structurally respectful theory in the language of sets and classes. However, this will also be a framework in which it is possible to use the rich combinatorial power afforded by material set theory in discussing notions of cardinality and structure.

Definition 9. The theory of Set Theory with Structures (or ZFCS) is defined as follows:

(i) Symbols:

(a) We have three sorts of variables: $u_{0}, u_{1}, \ldots, u_{n}, \ldots$ will range over urelements (to be featureless points in the domains of structures), $s_{0}, s_{1}, \ldots, s_{n}, \ldots$ will range over structures, and $x_{0}, x_{1}, \ldots, x_{n}, \ldots$ will range over sets.

(b) The usual logical symbols (so one's favourite connectives and quantfier(s)), and one non-logical symbol ' $\in$ ' (to denote material set membership).

(c) Symbols: A single symbol $U$ (for the universe), $f_{m, n}$ (for $m$-ary functions), $R_{m, n}$ (for $m$-ary relations) and $c_{n}$ (for constants), where $m, n$ are natural numbers and $m>0$. These will be used to describe structures.

(ii) Atomic formulas:

(a) $a=b$ where $a, b$ are variables of the same sort.

(b) $a \in b$ where $a$ is a variable and $b$ is a set-variable.

(c) $U(s, a)$ where $s$ is a structure-variable and $a$ is an urelement-variable. (Intended meaning: $a$ belongs to the universe (or domain) of the structure $s$.)

(d) $f_{m, n}\left(s, u_{1}, \ldots, u_{m}\right)=u$ where $s$ is a structure-variable, the $u_{i}$ and $u$ are urelement variables. (Intended meaning: the $u_{i}$ and $u$ belong to the universe of the structure $s$ and the interpretation of the $m$-ary function symbol $f_{m, n}$ in $s$ sends $\left(u_{1}, \ldots, u_{m}\right)$ to $u$.)

\footnotetext{
${ }^{30}$ See here, for example, [Shapiro, 1991].
} 
(e) $R_{m, n}\left(s, u_{1}, \ldots, u_{m}\right)$ where $s$ is a structure-variable and the $u_{i}$ are urelement variables. (Intended meaning: The $m$-tuple $\left(u_{1}, \ldots, u_{m}\right)$ belongs to the interpretation of the $m$-ary predicate symbol by the structure $s$.)

(f) $c_{n}(s)=u$ where $s$ is a structure-variable and $u$ is an urelement variable. (Intended meaning: The interpretation of the constant symbol $c_{n}$ by $s$ is $u$.)

(iii) Compound formulas: Obtained from atomic formulas by closing under connectives and quantifiers in the usual way. (Though, since the language is 3-sorted, there will be three kinds of quantifier; one for urelements, one for structures, and one for sets.)

(iv) Axioms:

(a) Extensionality for sets.

(b) Formula-Foundation for Sets: If a formula holds of some set then it holds of some set which is disjoint from all other sets for which the formula holds.

(c) The Axiom of Infinity: Usually rendered as concerning the existence of an inductive pure set.

(d) Pairing, Union, Powerset, Separation and Collection for sets.

(e) Axiom of Choice for sets.

(f) The domain of every structure is a set: i.e. $\forall s \exists x \forall a(U(s, a) \leftrightarrow a \in x)$.

(g) The Anti-Urelement Set Axiom: No set contains all of the urelements.

Some remarks concerning the definition are in order:

Remark 10. First, whilst the Anti-Urelement Set Axiom merits philosophical discussion 31 consideration in detail would take us too far afield here. We make this assumption simply to avoid bounding the sizes of the structures we have available, and settle for the pragmatic justification that we are trying to show that material set theory can convey important structural information, not that it must in every situation 32

Remark 11. Second, we are taking inspiration from the structuralist literature in the following sense: Structures are to be understood as composed of featureless points (given by the urelemente) and the ways they may correspond (with functions and relations). Effectively, we layer sets on top of antecedently given structures conceived of in this sense. As we'll see, this facilitates cardinality comparisons whilst allowing for a theory that respects isomorphism invariance.

\subsection{Class Theory with Structures (NBGS)}

We are now in a position where we have a theory of structures and the sets that can be built over them. In order to arrive at a language that respects isomorphism invariance we now augment with class variables. As we shall see, this allows us to latch onto a range of isomorphism invariant classes.

Definition 12. Our Class Theory with Structures (or NBGS) comprises the following:

1. Symbols: All the symbols of ZFCS, with an additional kind of variables $X_{0}, X_{1}, \ldots, X_{n}, \ldots$ for classes.

${ }^{31}$ For two examples of such discussion, see [McGee, 1997] and [Rumfitt, 2015].

${ }^{32} \mathrm{An}$ alternative would be to take the 'wide sets' approach of [Menzel, 2014] and modify Replacement. 
2. Atomic formulas: In addition to the well-formed formulas of ZFCS, we admit $X_{n}=X_{m}$ for class variables $X_{n}$ and $X_{m}$ as well-formed, as well as $v_{0} \in X_{n}$ for class-variable $X_{n}$ and $v_{0}$ is either a set, structure, or urelement variable.

3. Compound formulas: Obtained inductively from the connectives, $\in$, urelemente quantifiers, structure quantifiers, set quantifiers, and class quantifiers.

4. Axioms:

(a) All axioms of ZFCS.

(b) Extensionality for classes (i.e. $X_{n}$ and $X_{m}$ are equal iff they have the same members).

(c) Predicative Class Comprehension:

$$
\exists X \forall u \forall s \forall x[(\phi(u) \leftrightarrow u \in X) \wedge(\psi(s) \leftrightarrow s \in X) \wedge(\chi(x) \leftrightarrow x \in X)]
$$

(Where $u$ is a urelement variable, $s$ is a structure variable, and $x$ is a set variable, there are no class quantifiers ${ }^{33}$ in $\phi, \psi$, and $\chi$, and each of $\phi, \psi$, and $\chi$ is free for $u, s$, and $x$ respectively.)

Effectively we allow extensional classes composed of objects of mixed types. The intuition behind the theory is this; once we have built our sets and structures out of sets and urelements, we can then talk definably about them (much in the same way as it is legitimate to talk about definable classes in the ZFC context). As we'll shortly see, we can then restrict to certain classes in using our set and class-theoretic language to latch onto isomorphism invariant properties.

One technical issue is how to treat isomorphism within this framework. We really have two notions available:

Definition 13. Two structures $s_{0}$ and $s_{1}$ are structure-theoretically isomorphic iff there is a third structure $s$ within which there is a binary relation between the universes of $s_{0}$ and $s_{1}$ satisfying the usual rules of isomorphism.

Definition 14. Two structures $s_{0}$ and $s_{1}$ are set-theoretically isomorphic iff there is a set-theoretic bijection between the domains if $s_{0}$ and $s_{1}$ satisfying the usual rules of isomorphism.

Remark 15. Effectively the set-theoretic notion of isomorphism is the usual one, whilst the structure-theoretic notion pulls talk of structures and isomorphism into the prior given theory of structure.

What this prior given theory of structure satisfies will be important for our results and arguments, since we require the closure of our NBGS world under isomorphism. What we show below will always hold for the set-theoretic notion of isomorphism, but there is a chance that if the antecedent theory of structures over which we layer NBGS is too impoverished, then set-theoretic isomorphisms might not be mirrored by structure-theoretic ones. For example, consider a world which has NBGS layered over a theory of structure on which there is only one structure $s_{0}$ composed of an isolated point $u_{0}$ with no relations on it. This is be perfectly legitimate as a NBGS structure. However, while there are set-theoretic isomorphisms in

\footnotetext{
${ }^{33}$ We make this assumption merely for technical hygiene since NBGS will do the job we want neatly. One could also drop this restriction, and use an impredicative comprehension scheme yielding a structural form of Morse-Kelley (call it MKS). This may well have interesting additional properties, such as the ability to define a satisfaction predicate for the universe and first-order formulas.
} 
this world (specifically $f$ defined by the rule $f\left(u_{0}\right)=u_{0}$ ), the underlying theory of structure lacks the resources to even see that $s_{0}$ is isomorphic to itself.

One fix would be to introduce the following axiom:

Axiom 16. The Structural Richness Axiom: Any set-theoretic isomorphism has a corresponding extensionally equivalent structure-theoretic isomorphism 34

This would guarantee the existence of the isomorphisms we need for our results both set-theoretically and structure-theoretically. Indeed, we might derive the Structural Richness Axiom from the idea that any set-theoretic structure should be mirrored by a structure-theoretic one, postulating the following:

Axiom 17. The Structural Radical Richness Axiom: Any set-theoretic structure is mirrored by a corresponding structure-theoretic structure 35

The extent to which our theory of structure should mirror what we have going on in the sets is an interesting one, but we shall set it aside from our technical result. Since we wish to leave it entirely open what the underlying theory of structure should satisfy (maybe, for example, we just want to build NBGS over the structural groups and nothing else?) we shall simply concern ourselves for the technical work with the set-theoretic notion of isomorphism. Later, we will reconsider structural richness axioms in discussion how set theory has interesting things to say about the interaction of schematic types with structures.

We now have the resources to factor out non-isomorphism invariant properties from our theory whilst having combinatorial sets around to facilitate proof. This is shown by the following analogue of the earlier mentioned ETCS theorem concerning isomorphism invariance:

Theorem 18. (NBGS) Suppose that $\phi(v)$ is a formula without parameters in the language of NBGS and $v$ is a variable ranging over structures. Suppose that $\mathfrak{M}$ is a model for NBGS and $s_{0}$ and $s_{1}$ are structures in $\mathfrak{M}$ which are isomorphic in $\mathfrak{M}$. Then $\mathfrak{M} \models \phi\left(s_{0}\right) \leftrightarrow \phi\left(s_{1}\right)$.

Proof. The idea of the proof is simply to take an isomorphism $\pi$ between $s_{0}$ and $s_{1}$ and then use it to build an automorphism $\pi^{\prime}$ from $\mathfrak{M}$ to $\mathfrak{M}$, moving $\mathfrak{M}^{\prime}$ s satisfaction of $\phi\left(s_{0}\right)$ to $\phi\left(s_{1}\right)$ (dual reasoning obtains the converse implication). So, let $\pi$ be the witnessing structure-theoretic isomorphism. We define $\pi^{\prime}$ as follows:

(i) $\pi^{\prime}(u)=\pi(u)$ if $u$ is an urelement in $\operatorname{dom}(\pi)$.

(ii) $\pi^{\prime}(s)$ is obtained from $s$ by replacing each urelement $u$ in the universe of $s$ by $\pi(u)$.

(iii) We can then (by induction and the well-foundedness of membership) replace a set $x$ by defining $\pi^{\prime}(x)$ as $\left\{\pi^{\prime}(y) \mid y \in x\right\}$.

(iv) We similarly replace a class $X$ by $\pi^{\prime}(X)=_{d f}\left\{\pi^{\prime}(y) \mid y \in X\right\}$.

(v) This $\pi^{\prime}$ yields an automorphism of $\mathfrak{M}$ sending $s_{0}$ to $s_{1}$. Thus $\mathfrak{M}$ satisfies “ $\phi\left(s_{0}\right)$ iff $\phi\left(s_{1}\right)^{\prime \prime}{ }^{36}$

\footnotetext{
${ }^{34}$ In fuller formalism: If $f$ is a set-theoretic isomorphism between $s_{0}$ and $s_{1}$, then there is an $s$ such that $s$ maps $u_{\alpha}$ to $u_{\alpha}^{\prime}$ iff $f$ does.

${ }^{35}$ In fuller formalism: For any set $X$ of urelements and set-theoretic functions $f_{m, n}^{X}$, relations $R_{m, n}^{X}$ on $X$, and constants $c_{n}^{X}$ in $X$, there is an $s$ such that $U\left(s, u_{\alpha}\right)$ (for each $u_{\alpha} \in X$ ), and structural relations $f_{m, n}^{s}, R_{m, n}^{s}$, and $c_{n}^{s}$ equivalent to $f_{m, n}^{X}, R_{m, n}^{X}$, and $c_{n}^{X}$ in the obvious way.

${ }^{36}$ This can be proved by the usual tedious induction on the complexity of $\phi$.
} 
Remark 19. A very similar theorem holds for formulas with more free variables, providing that the domains of the structures are non-overlapping. In this way, NBGS can provide a kind of isomorphism invariance stronger than the one for ETCS as given in Theorem 8

Thus, while there are many non-isomorphism invariant facts we can state within NBGS (e.g. for an urelement or structure $v$, " $v \in X$ "), we can factor out this nonisomorphism invariant 'noise' in a precise way. Thus, for $\phi$ of the appropriate form, if we use the ambient material set-theoretic properties to prove $\phi$ about $s$, we can transfer $\phi$ to any structure isomorphic to $s$.

If we then allow ourselves some axiom of structural richness (e.g. either Axiom 16 or Axiom 17) we can talk about inter-structural relationships such as embeddability and cardinality of structures using set-theoretic resources, whilst factoring out the structural content. For example, concerning cardinality, every cardinal number exists, both with respect to the relevant set-theoretic code living in the universe of NBGS under consideration, but also there is (given an assumption of structural richness) a structure of pure cardinality (i.e. of featureless points with no relationships between them). In this way the theory provides a way of combinatorially linking bona fide structures with their set-theoretic codes.

This has ramifications for how we treat theorems best suited for material set theory. The Morley Categoricity Theorem, for example, can be recast as the claim that for any countable $\mathbf{T}$ in $\mathscr{L}_{\omega, \omega}$ (i.e. $\mathbf{T}$ is a particular kind of first-order schematic type) if $\mathbf{T}$ is satisfied by exactly one structure (up to isomorphism) for structures with domain of some particular uncountable size, then given any two structures satisfying $\mathbf{T}$, if their domains are uncountable and the same size, then they are isomorphic.

This version of the Morley Categoricity Theorem facilitates extraction of structural information: All the above can be recast as talk directly about theories and structures. However, the ambient material set theory provided by NBGS has a role to play: The proof could be formalised exactly as is usually done in the material settheoretic case, since we have the relevant set-theoretic resources, set-theoretic codes, and model theory lying around ${ }^{37}$ It is just that in the end we can easily extract the purely structural content from Morley's result using Theorem 18 and assumptions of structural richness, and then talk directly and transparently about structures conceived of in a sense independent of set-theoretic coding.

Moreover, not only do we now have a way to extract the purely structural information from the material set theory, but we can also find non-arbitrary representatives for structures:

Definition 20. In NBGS, we say that a class $X$ consisting of structures is (set- or structure-theoretically) invariant if $X$ is closed under isomorphism between structures. If in addition any two structures in $X$ are isomorphic we refer to $X$ as a (setor structure-theoretic) isomorphism type.

Thus, in NBGS we can equate specific mathematical structures with isomorphism types. This then gives us a non-arbitrary, non-coding dependent representative for the structure in question (since isomorphism types include all and only the structures isomorphic to one another). However, all this occurs in a framework where we have the full resources of material set theory available to speak about their relationships.

\footnotetext{
${ }^{37}$ See, for example, [Tent and Ziegler, 2012] for a presentation of the usual proof.
} 


\section{Conclusions and open questions}

How does this perspective provide philosophical progress? Our main claim (as specified in the introduction) was to show that material set theory seems to be interesting from the structural perspective. In particular, it still represents our best theory of cardinality, and how cardinality interacts with properties of syntactic theories. In this respect it is of interest to friends of both set-theoretic and category-theoretic foundations.

Of course one might simply reject that cardinality is a structural property, and that cardinality considerations fade away when one takes seriously a structural perspective as coded categorially ${ }^{38}$ This raises an important first question for future research:

Question 21. To what extent should cardinality be viewed as a structural property?

If we accept that cardinality is structural, however, it is interesting that we can, by taking much structural talk at face value, come up with a theory (namely NBGS) that allows us to easily extract structural content from set-theoretic claims. In this way, we have both the combinatorial power of set theory but also a way of factoring out non-isomorphism invariant content.

The theory represents a small first step on a wider research programme in the foundations of mathematics: How can we fuse different perspectives to yield new mathematical and philosophical perspectives and results? In particular, we have left open the following questions:

Question 22. There is the possibility of looking at categorial structure of theories like NBGS. Some, are easy: The category of isomorphism invariant classes that has as objects isomorphism types and equivalence classes of embeddings as arrows is an obvious choice. However, there is also the possibility of looking at the categorial structure of the classes of mixed type. What sorts of categorial relationships does this world support?

One remaining challenge is the following: In the introduction it was noted that certain categories have non-function-like relationships either for trivial reasons (such as the category of proofs of a logical system) or deeper ones concerning the nature of the category itself, such as with non-concrete categories like the homotopy category. The framework we have provided depends explicitly on a notion of structure on which isomorphism is understood as a functorial relationship between structures. A question then is:

Question 23. Can a similar perspective be found that brings set-theoretic combinatorial power to bear on non-concrete categories?

This would further help to inform the following project:

Question 24. Aside from the normal interpretations of category theory in set theory (either through universes or classes) or set theory in category theory (say via the use of objects and arrows coding membership-trees), what further ways are there of combining the different perspectives? We have shown how this can be done with respect to 'structure' and 'cardinality', but can we have further philosophically and mathematically fruitful fusions of these two perspectives?

For the moment, we hope to have shown that there is at least the possibility of combining different perspectives to yield interesting fusions of foundational theories.

${ }^{38}$ We are grateful to Steve Vickers for pressing this point. 


\section{References}

[Andrés Eduardo Caicedo, 2017] Andrés Eduardo Caicedo, James Cummings, P. K. P. B. L., editor (2017). Foundations of Mathematics: Logic at Harvard Essays in Honor of W. Hugh Woodin's 60th Birthday, volume 690 of Contemporary Mathematics. American Mathematical Society.

[Antos et al., S] Antos, C., Barton, N., and Friedman, S.-D. (S). Universism and extensions of $V$. Submitted.

[ASL, 2014] ASL (2014). 2013 north american annual meeting of the association for symbolic logic: University of waterloo waterloo, ontario, canada may 8-11, 2013. The Bulletin of Symbolic Logic, 20(1):105-133.

[Awodey, 1996] Awodey, S. (1996). Structure in mathematics and logic: A categorical perspective. Philosophia Mathematica, 4(3):209-237.

[Bagaria and Brooke-Taylor, 2013] Bagaria, J. and Brooke-Taylor, A. (2013). On colimits and elementary embeddings. The Journal of Symbolic Logic, 78(2):562-578.

[Baldwin, 2018] Baldwin, J. T. (2018). Model Theory and the Philosophy of Mathematical Practice: Formalization without Foundationalism. Cambridge University Press.

[Barton, 2016] Barton, N. (2016). Multiversism and Concepts of Set: How Much Relativism Is Acceptable?, pages 189-209. Springer International Publishing, Cham.

[Barton, S] Barton, N. (S). Forcing and the universe of sets: Must we lose insight? Submitted.

[Bell, 2011] Bell, J. (2011). Set Theory: Boolean-Valued Models and Independence Proofs. Oxford University Press.

[Benacerraf, 1965] Benacerraf, P. (1965). What numbers could not be. Philosophical Review, 74(1):47-73.

[Ernst, 2015] Ernst, M. (2015). The prospects of unlimited category theory: Doing what remains to be done. The Review of Symbolic Logic, 8(2):306-327.

[Ernst, 2017] Ernst, M. (2017). Category theory and foundations. In Landry, E., editor, Landry, 2017], pages 69-89. Oxford University Press.

[Ewald, 1996] Ewald, W. B., editor (1996). From Kant to Hilbert. A Source Book in the Foundations of Mathematics, volume I. Oxford University Press.

[Feferman, 1977] Feferman, S. (1977). Categorical foundations and foundations of category theory. In Butts, R. E. and Hintikka, J., editors, Logic, Foundations of Mathematics, and Computability Theory, pages 149-169. Springer.

[Freyd, 1970] Freyd, P. (1970). Homotopy is not concrete, pages 25-34. Springer Berlin Heidelberg, Berlin, Heidelberg.

[Goldblatt, 1984] Goldblatt, R. (1984). Topoi: The Categorial Analysis of Logic. Dover Publications.

[Hamkins, 2012] Hamkins, J. D. (2012). The set-theoretic multiverse. The Review of Symbolic Logic, 5(3):416-449. 
[Hellman, 2006] Hellman, G. (2006). Against 'absolutely everything'! In Rayo, A. and Uzquiano, G., editors, Absolute Generality. Clarendon Press.

[Isaacson, 2011] Isaacson, D. (2011). The reality of mathematics and the case of set theory. In Noviak, Z. and Simonyi, A., editors, Truth, Reference, and Realism, pages 1-75. Central European University Press.

[Landry, 2017] Landry, E., editor (2017). Categories for the Working Philosopher. Oxford University Press.

[Landry and Marquis, 2005] Landry, E. and Marquis, J.-P. (2005). Categories in context: Historical, foundational, and philosophical $\dagger$. Philosophia Mathematica, 13(1):1.

[Lawvere, 1965] Lawvere, W. (1965). An elementary theory of the category of sets. Proceedings of the National Academy of Science of the U.S.A., 52:1506-1511.

[Lawvere and McLarty, 2005] Lawvere, W. and McLarty, C. (2005). An elementary theory of the category of sets (long version) with commentary. In Reprints in Theory and Applications of Categories 11, pages 1-35. TAC.

[Leinster, 2014] Leinster, T. (2014). Rethinking set theory. The American Mathematical Monthly, 121(5):403-415.

[Mac Lane, 1971] Mac Lane, S. (1971). Categorical algebra and set-theoretical foundations. In [Scott and Jech, 1971], pages 231-240. American Mathematical Society.

[Mac Lane, 1986] Mac Lane, S. (1986). Mathematics: Form and Function. SpringerVerlag.

[Maddy, 2017] Maddy, P. (2017). Set-theoretic foundations. In Andrés Eduardo Caicedo, 2017], pages 289-322. American Mathematical Society.

[Maddy, F] Maddy, P. (F). What do we want a foundation to do? In Reflections on the Foundations of Mathematics: Univalent Foundations, Set Theory and General Thoughts. Springer.

[Makkai, 1998] Makkai, M. (1998). Towards a categorical foundation of mathematics. In Lecture Notes in Logic, volume 11, pages 153-190. Springer.

[Marquis, 2017] Marquis, J.-P. (2017). Unfolding FOLDS: A foundational framework for abstract mathematical concepts. In Landry, E., editor, [Landry, 2017], pages 136-162. Oxford University Press.

[Mathias, 2001] Mathias, A. (2001). The strength of mac lane set theory. Annals of Pure and Applied Logic, 110(1):107 - 234.

[Mathias, 2000] Mathias, A. R. D. (2000). Strong statements of analysis. Bulletin of the London Mathematical Society, 32(5):513-526.

[McGee, 1997] McGee, V. (1997). How we learn mathematical language. The Philosophical Review, 106(1):35-68.

[McLarty, 1993] McLarty, C. (1993). Numbers can be just what they have to. Noûs, 27(4):487-498.

[Mclarty, 2004] Mclarty, C. (2004). Exploring categorical structuralism. Philosophia Mathematica, 12(1):37-53. 
[Meadows, 2013] Meadows, T. (2013). What can a categoricity theorem tell us? The Review of Symbolic Logic, 6:524-544.

[Menzel, 2014] Menzel, C. (2014). $Z F C U$, wide sets, and the iterative conception. Journal of Philosophy, 111(2):57-83.

[Muller, 2001] Muller, F. A. (2001). Sets, classes and categories. British Journal for the Philosophy of Science, 52:539-573.

[Osius, 1974] Osius, G. (1974). Categorical set theory: A characterization of the category of sets. Journal of Pure and Applied Algebra, 4(1):79 - 119.

[Rumfitt, 2015] Rumfitt, I. (2015). The Boundary Stones of Thought: An Essay in the Philosophy of Logic. Oxford University Press.

[Scott and Jech, 1971] Scott, D. and Jech, T. (1971). Axiomatic Set Theory. Number pt. 1 in Axiomatic Set Theory. American Mathematical Society.

[Shapiro, 1991] Shapiro, S. (1991). Foundations without Foundationalism: A Case for Second-order Logic. Oxford University Press.

[Shepherdson, 1951] Shepherdson, J. C. (1951). Inner models for set theory-part i. Journal of Symbolic Logic, 16(3):161-190.

[Shepherdson, 1952] Shepherdson, J. C. (1952). Inner models for set theory-part ii. Journal of Symbolic Logic, 17(4):225-237.

[Shulman, 2010] Shulman, M. A. (2010). Stack semantics and the comparison of material and structural set theories.

[Tent and Ziegler, 2012] Tent, K. and Ziegler, M. (2012). A Course in Model Theory. Lecture Notes in Logic. Cambridge University Press.

[The Univalent Foundations Program, 2013] The Univalent Foundations Program (2013). Homotopy Type Theory: Univalent Foundations of Mathematics. http: //homotopytypetheory.org/book.

[Tsementzis, 2016] Tsementzis, D. (2016). Univalent foundations as structuralist foundations. Synthese.

[Zermelo, 1930] Zermelo, E. (1930). On boundary numbers and domains of sets. In [Ewald, 1996], volume 2, pages 1208-1233. Oxford University Press. 